3

4

5

6

7

8

9

10

11

\title{
Quantifying parameters of bottlenose dolphin
} signature whistles

\author{
H. Carter Esch
}

Woods Hole Oceanographic Institution

Biology Department, M.S. \#33

Woods Hole, MA 02543, USA

E-mail: carter@whoi.edu

Laela S. Sayigh

Woods Hole Oceanographic Institution

Biology Department, M.S. \#50

Woods Hole, MA 02543, USA

E-mail: lsayigh@whoi.edu

Randall S. Wells

Chicago Zoological Society

c/o Mote Marine Laboratory

Sarasota, FL 34236, USA

rwells@mote.org 
Bottlenose dolphins (Tursiops truncatus) produce individually distinctive

vocalizations called signature whistles, first described by Melba and David Caldwell (1965). The Caldwells observed that isolated, captive dolphins produced whistles with individually distinctive frequency contours, or patterns of frequency changes over time, and hypothesized that these whistles were used to transmit identity information (Caldwell and Caldwell 1965; Caldwell et al. 1990). Since the Caldwell’s work with isolated, captive dolphins, several studies have documented signature whistles in a variety of contexts, including free-swimming captive dolphins (e.g., Janik and Slater 1998; Tyack 1986), briefly restrained wild dolphins (e.g., Sayigh et al. 1990, 2007, Watwood et al. 2005), and free-ranging wild dolphins (e.g., Watwood 2003; Watwood et al. 2004, 2005; Buckstaff 2004; Cook et al. 2004). Janik and Slater (1998) demonstrated that signature whistles are used to maintain group cohesion, thus supporting the Caldwells' hypothesis. Janik et al. (2006) verified experimentally that bottlenose dolphins respond to signature whistles produced by familiar conspecifics even after voice featured have been removed, reinforcing the notion that the contour of a signature whistle carries identity information.

Signature whistle parameters vary by age (Caldwell et al. 1990; Esch et al. in press), sex (Sayigh et al. 1995, Esch et al. in press), and context (Caldwell et al. 1990; Janik et al. 1994; Watwood et al. 2005; Esch et al. in press). Young dolphins (both male and female) have higher signature whistle rates than adults, but whistle rate decreases more quickly with age in males than females (Caldwell et al. 1990, Esch et al. in press). Adult dolphins produce more loops per whistle (and therefore longer whistles) than infants and 
sub-adults (Caldwell et al. 1990). Caldwell et al. (1990) found that certain parameters of signature whistles (e.g., frequency, number of loops and duration of loops) appeared to be closely related to the level of arousal of an individual dolphin; however, these differences were not consistent across individuals. Esch et al. (in press) found that whistle rate and the number of loops produced per whistle varied by context, and hypothesized that increases in these whistle parameters may be indicative of stress in bottlenose dolphins. Similarly, Janik et al. (1994) found that 9 of 14 signature whistle frequency and time parameters differed significantly between isolation and interaction conditions, supporting the existence of both identity and context related information in signature whistles. However, despite this variability in an individual dolphin's signature whistle parameters, the overall contour usually remains highly stereotyped for at least a decade (Caldwell et al. 1990; Sayigh et al. 1990; Janik and Slater 1994; Esch et al. in press).

8 e.g., FB24, FB35, Figure 1), or variable numbers of repeated loops, which may or may not be connected (e.g., connected, FB20, FB118; disconnected, FB138, FB220, Figure 1). Some multi-looped whistles also contain an introductory and/or terminal loop, which differ in contour from the central loops (e.g., FB48, FB97, Figure 1; Caldwell et al. 1973, 1990, Sayigh et al. 1990). For whistles with multiple disconnected loops some studies have considered each loop repetition as a separate whistle (e.g., Schevill and Watkins 1962; Tavolga 1968; McCowan and Reiss 2001), while others have distinguished loops from whistles (e.g., Caldwell et al. 1973, 1990, Sayigh et al. 1990, 2007; Buckstaff 2004; 
Watwood 2003; Watwood et al. 2005; Esch et al. in press). In the present study, we hypothesized that loops are separated by highly stereotyped time intervals, and that stereotyped loops and silences between loops both play a part in the production of a unique signal (based on Caldwell et al. 1990). The presence of an introductory and/or terminal loop (e.g., Figure 1: FB25, FB48, FB54, FB84, FB97, and FB220) supports the idea that multiple disconnected loops should be considered part of the same unit if separated by stereotyped silences (Caldwell et al. 1973, 1990). It is important that studies of dolphin communication are consistent in how multi-looped whistles are treated; otherwise studies that include this type of signal are difficult to compare. Thus, a goal of this study was to quantify inter-loop intervals in stereotyped sequences of disconnected loops, in order to test the hypothesis that these intervals are shorter and more consistent (less variable) than are the intervals between successive whistles.

A second goal of this study was to quantify the acoustic parameters of signature whistles (especially maximum frequency, but measurements were also made of minimum frequency, and overall duration) to update the documented ranges of these values. Many studies of dolphin signature whistles utilized recording equipment with upper frequency cut-offs at or below $24 \mathrm{kHz}$, and were thus unable to measure higher frequencies (e.g., Azevedo and Oliveira 2007, Dreher 1961, Evans and Prescott 1962, Sayigh et al. 1990, Steiner 1981, Tyack 1986, Wang et al. 1995). Currently, the value of $24 \mathrm{kHz}$ reported by Caldwell et al. (1990) is the highest maximum frequency for signature whistles in the 
literature. We report values for the fundamental frequency of signature whistles and do not include harmonics or other types of vocalizations (e.g., echolocation).

\section{Recordings of long-term resident bottlenose dolphins from brief capture-release} events in Sarasota Bay, Florida (Scott et al. 1990; Wells 1991, 2003; Wells et al. 2004), have been collected over a period of 34 years (1975-2008), and many dolphins have been recorded multiple times (maximum $=15$, mean $=3.3$ ). Custom-built suction cup hydrophones were placed directly on the head of each individual, allowing researchers to unequivocally identify the vocalizing dolphin. The hydrophones were developed and built at the Woods Hole Oceanographic Institution (WHOI; circuitry described in Tyack 1985), and were equipped with 1-2 kHz high-pass filters, above which their frequency response was flat to $25 \mathrm{kHz}$. The hydrophones were not calibrated because amplitude values were not being measured. Whistles were recorded onto either Marantz PMD-430 or Sony TCD5M stereo-cassette recorders (frequency response »30-20000 Hz, digitization sampling rate 96 kHz, 24bit), Panasonic AG-6400 or AG-7400 video-cassette recorders (frequency response »20-32000 Hz, digitization sampling rate $96 \mathrm{kHz}$, 24bit), or a Sound Devices 744-T digital recorder (frequency response 10-48000 Hz, sampling rate 96 kHz, 24 bit). The predominant whistle produced by an animal during a brief capture-release event is defined as its signature whistle. Other whistles produced during these recording sessions are called non-signature whistles. The Sarasota Dolphin Community Signature Whistle Catalogue (Sayigh, unpublished data) currently contains signature whistles from 205 dolphins. Since most dolphins in Sarasota Bay have been captured and released more than 
once, signature whistle identifications for all dolphins included in this study have been confirmed by reviewing multiple recordings for an individual animal.

Twenty whistles produced by each of 28 different dolphins (12 male, 16 female) were randomly selected from all whistles produced by an individual dolphin in a single recording session during brief capture-release events between 1988 and 2001 in Sarasota Bay, FL. These randomly selected whistles were primarily signatures, but in some cases non-signatures were selected. Dolphins were chosen so a variety of different types of signature whistle were represented, including:

1. Loops sometimes connected, sometimes not; may vary in number and/or contour (4 of 28 dolphins; e.g., FB146, FB151, FB166, FB186, Figure 1);

2. Loops always disconnected, may vary in number and/or contour (14 of 28 dolphins; e.g., FB7, FB9, FB11, FB25, FB38, FB48, FB54, FB55, FB84, FB90, FB97, FB101, FB138, FB220. Figure 1);

3. Loops always connected, may vary in number and/or contour (8 of 28 dolphins; e.g., FB3, FB20, FB67, FB105, FB118, FB122, FB140, FB163, Figure 1);

4. No repetitive loop structure (2 of 28 dolphins; e.g., FB24, FB35, Figure 1). In the recording library of 205 dolphins used as a resource in this study, the four whistle types listed above were represented as follows: type 1, 4.3\%, type 2, 39.9\%, type 3, 33.7\%, and type $4,22.1 \%$. 
A continuous whistle was classified as multi-looped (i.e., consisting of multiple

134

135

136

137

138

139

140

141

connected repeated elements) based on previous visual classification of a large dataset of whistles by human judges (Sayigh et al. 2007). To develop a criterion for classifying whistle elements as disconnected loops or as separate whistles, inter-element intervals were measured during 30 min of a recording for each of 5 dolphins (FB2, FB15, FB33, FB38, FB101). None of these recordings were included in the data set used for later analyses. The mean number of whistle elements in these recordings was $461 \pm 315$. Individual elements were assigned to a single whistle (i.e., a whistle with multiple disconnected loops) using the criterion defined by Janik and Slater (1998): elements separated by 0.5 seconds or less were considered loops in a single whistle. Whistle classification using this criterion agreed with visual classification in all cases (Table 1); therefore, this criterion (i.e., elements that occurred within 0.5 sec of each other) was used to classify whistle elements as loops vs. separate whistles in the current study.

When possible, a single recording session for each dolphin was analyzed utilizing Signal/RTSD (Version 3.0, Engineering Design, Belmont, MA) or Avisoft-SASLab Pro 3.2 (Raimund Specht, Berlin, Germany), which are software packages that display realtime spectrograms. Every whistle produced during the chosen recording session was noted and numbered, with a minimum sample size of 200 whistles for each dolphin. In six cases, 200 whistles did not occur in the recording session chosen. In these cases, an additional session was also analyzed in order to reach a minimum of 200 whistles. Sample sizes ranged from 201 to 2,144 whistles per dolphin (mean $=308 \pm 416)$. A table of 20 random 
numbers was generated (in Microsoft Excel) for each dolphin, based on its total quantity of whistles. These 20 randomly selected whistles were then subjected to further analyses. For six dolphins in this study, non-signature whistles were present in the random sample; however, parameter measurements for signature and non-signature whistles are presented separately. Only signature whistles were included in inter-loop and inter-whistle interval comparisons.

Inter-loop intervals can be distinguished from inter-whistle intervals on the basis of significant differences in duration and variability. Inter-loop intervals in stereotyped sequences of disconnected loops were significantly shorter (Table 2, mean inter-loop interval $=0.10 \mathrm{~s}$, mean inter-whistle interval $=17.1 \mathrm{~s}$; paired t-test, $\mathrm{df}=15, P=0.01)$ and less variable (F-test, Table 2) than intervals between successive whistles. Standard deviations ranged from 0.01 to $0.06 \mathrm{sec}$ for inter-loop intervals versus 1.74 to $163.17 \mathrm{~s}$ for inter-whistle intervals. Coefficients of variation (CV, calculated as the ratio of standard deviation to the mean) ranged from 0.09 to 0.77 for inter-loop intervals versus 0.63 to 2.34 for inter-whistle intervals. Inter-loop interval values were more normally distributed while inter-whistle interval values were logarithmically distributed (Figure $2 \mathrm{a}, \mathrm{b}$ ). This difference should be even more pronounced in contexts other than capture-release, when whistle rates are much lower (i.e., inter-whistle intervals are longer; Esch et al. in press). These different distributions and resulting difference in variances between the two groups support the conclusion that inter-loop intervals are significantly less variable than interwhistle intervals, and may be an important component of signature whistle stereotypy. 
Means, standard deviations, and CV values for frequency maxima and minima, and

duration of each dolphin's signature whistle are presented in Table 3. Values for the 12 non-signature whistles included in the random sample are also shown. Mean maximum frequencies for signature whistles ranged from 9.3 to $27.3 \mathrm{kHz}$, with the latter exceeding the published upper range for bottlenose dolphin signature whistles ( $24 \mathrm{kHz}$, Caldwell et al. 1990; 17.8 kHz, Janik et al. 1994; 23.48 kHz, Buckstaff 2004). Mean minimum frequencies for signature whistles ranged from 3 to $13.3 \mathrm{kHz}$, and durations ranged from $0.5-2.3 \mathrm{~s}$, similar to values reported in other studies.

These results indicate that signature whistles have a greater range of frequencies than was previously reported, due to the increased maximum frequency value presented here. Variability in maximum or minimum frequencies may be caused by an introductory or terminal loop, such as a final upsweep or downsweep that tails off at a different frequency from one whistle to another (e.g., FB48, FB54, FB97, FB105, Figure 1). Coefficients of variation were often higher for dolphins that produced signature whistles with a variable introductory or terminal loop (Figure 1, Table 3, FB48, FB54, FB97, FB105). While several dolphins showed higher CV values for maximum than minimum frequency (Table 3, FB25, FB105), others showed the reverse pattern (Table 3, FB55, FB90, FB122). Thus, perhaps one frequency parameter (maximum or minimum) plays a more consistent role in signature whistle stereotypy in a given individual. 
Bottlenose dolphin whistle parameters have been reported in multiple studies,

200

201

202

203

204

205

206

207

208

209

210

211

212

213

214

215

216

217

218

219

220

although few studies distinguish between signature and non-signature whistles. With the exception of maximum frequency, our findings fall within previously published ranges.

Caldwell et al. (1990) reported maximum frequencies for bottlenose dolphin signature whistles ranging from $8-24 \mathrm{kHz}$, with minimum frequencies ranging from $1-9 \mathrm{kHz}$.

Signature whistle duration ranged from 0.2 - 2.1 s (Caldwell et al. 1990). Janik et al. (1994) documented signature whistle parameters for a single captive bottlenose dolphin in multiple contexts (minimum frequency: $4 \mathrm{kHz}$, maximum frequency: $17.8 \mathrm{kHz}$, duration range: 0.13 - $0.18 \mathrm{~s})$. Buckstaff (2004) reported signature whistle parameters for dolphins in Sarasota Bay, Florida, as part of a study on the effects of watercraft activity on acoustic behavior (frequency range: $2.91-23.48 \mathrm{kHz}$, duration range: $0.10-4.11 \mathrm{~s}$ ). Wang et al. (1995) determined whistle (combined signature and non-signature) parameters for bottlenose dolphins in Argentina, reporting frequencies ranging from $1.17-21.6 \mathrm{kHz}$, and a mean duration of $1.14 \mathrm{~s}$. Azevedo and Oliviero (2007) documented characteristics of whistles from a resident population of bottlenose dolphins in southern Brazil (minimum frequency range: 1.2 - $17.2 \mathrm{kHz}$, maximum frequency range: $3.6-22.3 \mathrm{kHz}$, duration range: $0.048-2.458 \mathrm{~s}$ ). Finally, in a recent study of geographic variation in bottlenose dolphin whistles (combined signature and non-signature), May-Collado and Wartzok (2008) provide an extensive review of whistle parameters for bottlenose dolphins in the Atlantic (minimum frequency range: $1.6 \mathrm{kHz}-18.92 \mathrm{kHz}$, maximum frequency range: 1.7 $\mathrm{kHz}-28.48 \mathrm{kHz}$, duration range: 0.005 - $1.3 \mathrm{~s}$ ). May-Collado and Wartzok (2008) report a higher maximum frequency than our study; however, our study focuses only on signature 
whistles while May-Collado and Wartzok (2008) do not distinguish among whistle types. Therefore, our study is the first to extend the frequency range of signature whistles above $24 \mathrm{kHz}$.

Caldwell et al. (1990) were the first to suggest that "rather than repeating a constant section of whistle, dolphins[s] [may] repeat both a section of whistle and an interval of silence”, and that those intervals may be highly consistent (although inter-loop interval values were not presented in their study). Our results indicate that inter-loop intervals can be quantitatively distinguished from inter-whistle intervals, and that inter-loop durations are much more consistent than inter-whistle durations for dolphins that produced multiple disconnected loops. While variations in frequency contour provide one mechanism for creating an individually distinctive whistle, the possible conformations are finite. For whistles with multiple disconnected loops, the stereotyped silence between loops may serve as another characteristic by which individual dolphins can distinguish themselves uniquely. In addition, the presence of a characteristic introductory or terminal loop in some signature whistles implies that the series of elements is produced as a punctuated unit. The results of this study indicate that it is appropriate to consider these loops as components of a single whistle, rather than as separate whistles. 
246 We would like to thank Dolphin Quest, the National Marine Fisheries Service, Mote

247 Marine Laboratory, and the Chicago Zoological Society for support of capture-release

248 operations. Thanks to the dedicated staff, students, and volunteers whose efforts ensured

249 the success and safety of the capture-release operations. We would also like to thank three

250 anonymous reviewers, whose suggestions greatly improved this manuscript. This work

251 was funded by a Protect Wild Dolphins grant from the Harbor Branch Oceanographic

252 Institution, issued to LSS and RSW. The research was conducted under NMFS Scientific

253 Research Permits No. 417, 655, 945, and 522-1569 issued to RSW, and approvals by the

254 Institutional Animal Care and Use Committees of both Mote Marine Laboratory and the

255 University of North Carolina Wilmington.

256

257

258

259

260

261

262 
265 Azevedo, A.F., and A.M. OliveirA. 2007. Characteristics of whistles from

266 resident bottlenose dolphins (Tursiops truncatus) in southern Brazil.

267 Journal of the Acoustical Society of America 121:2978-2983.

268

BUCKSTAFF, K.C. 2004. Effects of watercraft noise on the acoustic behavior of bottlenose dolphins, Tursiops truncatus, in Sarasota Bay, Florida. Marine Mammal Science 20:709-725.

272

273 CALDWELL, M.C., and D.K. CALDWELL. 1965. Individual whistle contours in 274 bottlenosed dolphins (Tursiops truncatus). Nature 207:434-435.

275

276

277 individual signature whistles in the spotted dolphin, Stenella plagiodon. Cetology 16:127821.

279

280 Caldwell, M.C., D.K. Caldwell, and P.L. TyaCK. 1990. Review of the 281 signature-whistle hypothesis for the Atlantic bottlenose dolphin. Pages 199-234 in S. 282 Leatherwood and R.R. Reeves, eds. The Bottlenose Dolphin. Academic Press, New 283 York, NY. 
285 CoOK, M.L.H., L.S. SAYIGH, J.E. Blum, and R.S. WeLLS. 2004. Signature whistle

286 production in undisturbed free-ranging bottlenose dolphins (Tursiops truncatus).

287 Proceedings of the Royal Society, Series B271:1043-1049.

288

DREHER, J.J. 1961. Linguistic considerations of porpoise sounds. Journal of the Acoustical Society of America 33:1799-1800.

EsCH, H.C., L.S. SAYIGH, J.E. BLuM, and R.S. WELlS. in press. Whistles as potential indicators of stress in bottlenose dolphins (Tursiops truncatus). Journal of Mammology.

Evans, W.E., and J.H. PrescotT. 1962. Observations of the sound production capabilities of the bottlenosed porpoise: A study of whistles and clicks. Zoologica 47:121-128.

298

JANIK, V.M. and P.J.B. SLATER. 1998. Context-specific use suggests that bottlenose dolphin signature whistles are cohesion calls. Animal Behavior 56:829-838.

JANIK, V.M., L.S. SAYIGH, and R.S. WELLS. 2006. Signature whistle shape conveys 
identity information to bottlenose dolphins. Proceedings of the National Academy of Sciences 103:8293-8297.

309 Mammalogy 89:1229-1240.

313

SAYigh, L.S., P.L. TyACK, R.S WelLS, M.D. SCOTT, and A.B. IRVINE. 1995. Sex differences in signature whistle production of free-ranging bottlenose dolphins, Tursiops truncatus. Behavioral Ecology and Sociobiology 36:171-177.

SAYIGH, L. S., H.C. EsCH, R.S. WELLS, and V.M. JANIK. 2007. Facts about signature whistles of bottlenose dolphins, Tursiops truncatus. Animal Behavior 74:16311642. 
330 ScheVILL, W.E., and W.A. WAtKIns. 1962. "Whale and Porpoise Voices.” Woods

331 Hole Oceanographic Institution, Woods Hole, Massachusetts. Phonograph record and 24-

332 page booklet.

333

334 ScotT, M.D., R.S. WelLS, and A.B. IRVINE. 1990. A long-term study of bottlenose

335 dolphins on the west coast of Florida. Pp. 235-244 in The Bottlenose Dolphin (S.

336 Leatherwood and R.R. Reeves, eds.). Academic Press, New York.

337

338

339

340

STEINER, W. 1981. Species-specific differences in pure tonal whistle vocalizations of five western North Atlantic dolphin species. Behavioral Ecology and Sociobiology 9:241-246.

341

342

TAVOLGA, W.N. 1968. Marine animal data atlas. Naval Training Device Center, Tech.

344 Rep., NAVTRADEVCEN 1212-2:i-x, 1-239.

TYACK, P.L. 1985. An optical telemetry device to identify which dolphin produces a sound. Journal of the Acoustical Society of America 78:1892-1895.

TyACK, P.L. 1986. Whistle repertoires of two bottlenosed dolphins, Tursiops truncatus: Mimicry of signature whistles? Behavioral Ecology and Sociobiology 18:251-257. 
352 WANG, D., WÜrsig, B., and EvANS, W.E. 1995. Whistles of bottlenose dolphins:

353 Comparisons among populations. Aquatic Mammals 21:65-77.

354

WATwOOD, S.L. 2003. Whistle use and whistle sharing by allied male bottlenose dolphins, Tursiops truncatus. Ph.D. thesis, Woods Hole. 227 pp. truncatus. Animal Behavior 69:1373-1386.

365

WATWOOD, S.L., P.L. TYACK, and R.S. WeLLS. 2004. Whistle sharing in paired male bottlenose dolphins, Tursiops truncatus. Behavioral Ecology and Sociobiology 55:531543.

WATwood, S.L., E.C.G. Owen, P.L. TyACK, and R.S. Wells. 2005. Signature whistle use by temporarily restrained and free-swimming bottlenose dolphins, Tursiops

WELLS, R.S. 1991. The role of long-term study in understanding the social structure of a bottlenose dolphin community. Pages 199-225 in K. Pryor and K.S. Norris, eds. Dolphin Societies: Discoveries and Puzzles. Univ. of California Press, Berkeley, CA.

WELLS, R.S. 2003. Dolphin social complexity: Lessons from long-term study and life history. Pages 32-56 in F.B.M. de Waal and P.L. Tyack, eds. Animal Social Complexity: Intelligence, Culture, and Individualized Societies. Harvard University Press, 
373

374

375

376

377

378

379

380

381

382

383

384

385

386

387

388

389

390

391

392

393

394

395
Cambridge, MA.

Wells, R.S., H.L. Rhinehart, L.J. HANSEn, J.C. SweEney, F.I. TOWNSEND, R.

Stone, D. Casper, M.D. ScotT, A.A. Hohn, and T.K. Rowles. 2004.

Bottlenose dolphins as marine ecosystem sentinels: Developing a health monitoring system. EcoHealth 1:246-254.

(1)

(1)

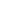

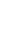

(1)


396

397

398

399

400

401

402

403

404

405

406

407

408

409

410

411

412

413

414

415

Figure 1. Spectrograms of the signature whistle for each of 28 dolphins. Frequency $(\mathrm{kHz})$ is on the $y$-axis and time (s) is on the $\mathrm{x}$-axis. Identical time and frequency scaling was used among all signature whistle exemplars.

Figure $2(a, b)$. Inter-loop $(n=521)$ and inter-whistle $(n=290)$ interval distributions. Intervals are shown in seconds (note different scales).

Table 1. Results of transition matrix (TM) and visual classifications (VC) of disconnected element whistle membership.

Table 2. Mean \pm SD (CV) inter-loop and inter-whistle durations(s) for each dolphin. CV values were calculated as the ratio of the SD to the mean. F-tests comparing inter-loop and inter-whistle variance values were all significant at $P<0.001$.

Table 3. Means, standard deviations (SD), and coefficients of variation (CV) for 20 whistles from each of the 28 dolphins. Non-signature whistle values are shown for six dolphins for which the 20 randomly selected whistles included non-signature whistles $(*)$. 
416

\begin{tabular}{|c|c|c|c|}
\hline Animal & \# of elements & \# of whistles: TM & \# of whistles: VC \\
\hline FB2 & 862 & 442 & 442 \\
\hline FB15 & 641 & 319 & 319 \\
\hline FB33 & 396 & 137 & 137 \\
\hline FB38 & 64 & 28 & 28 \\
\hline FB101 & 340 & 147 & 147 \\
\hline
\end{tabular}

417

418 Table 1

419

420

421

422

423

424

425

426

427

428

429

430

431

432

433

434

435 
Inter-loop duration Inter-whistle duration

\begin{tabular}{|c|c|c|c|}
\hline Animal & $\pm \mathrm{SD}(\mathrm{CV})$ & $\pm \mathrm{SD}(\mathrm{CV})$ & $\mathrm{F}$ \\
\hline FB7 & $0.12 \pm 0.01(0.09)$ & $15.40 \pm 12.21(0.79)$ & $1.4 \times 10^{6}$ \\
\hline FB9 & $0.13 \pm 0.04(0.31)$ & $8.09 \pm 10.96(1.35)$ & $7.5 \times 10^{4}$ \\
\hline FB11 & $0.07 \pm 0.02(0.31)$ & $8.14 \pm 11.02(1.35)$ & $3.0 \times 10^{4}$ \\
\hline FB25 & $0.07 \pm 0.02(0.30)$ & $6.28 \pm 14.91(2.26)$ & $5.2 \times 10^{4}$ \\
\hline FB38 & $0.09 \pm 0.01(0.11)$ & $10.76 \pm 10.97(0.98)$ & $1.2 \times 10^{6}$ \\
\hline FB48 & $0.05 \pm 0.01(0.19)$ & $29.33 \pm 26.15(0.89)$ & $6.8 \times 10^{6}$ \\
\hline FB54 & $0.09 \pm 0.04(0.46)$ & $35.08 \pm 60.49(1.72)$ & $2.3 \times 106$ \\
\hline FB55 & $0.19 \pm 0.03(0.14)$ & $13.63 \pm 14.31(1.05)$ & $2.3 \times 10^{5}$ \\
\hline & & $107.24 \pm 163.17$ & $2.7 \times 10^{8}$ \\
\hline FB84 & $0.11 \pm 0.01(0.12)$ & $(1.52)$ & \\
\hline FB90 & $0.10 \pm 0.03(0.27)$ & $6.34 \pm 6.99(1.10)$ & $5.4 \times 10^{4}$ \\
\hline FB97 & $0.07 \pm 0.01(0.13)$ & $6.79 \pm 15.88(2.34)$ & $2.5 \times 10^{6}$ \\
\hline FB101 & $0.23 \pm 0.06(0.24)$ & $11.92 \pm 18.59(1.56)$ & $5.9 \times 10^{4}$ \\
\hline FB138 & $0.11 \pm 0.01(0.09)$ & $2.75 \pm 1.74(0.63)$ & $3.7 \times 10^{2}$ \\
\hline FB146 & $0.06 \pm 0.02(0.35)$ & $3.41 \pm 2.66(0.78)$ & $5.8 \times 10^{1}$ \\
\hline FB166 & $0.07 \pm 0.01(0.14)$ & $5.25 \pm 3.86(0.74)$ & $7.6 \times 10^{2}$ \\
\hline FB220 & $0.09 \pm 0.01(0.13)$ & $2.95 \pm 2.59(0.88)$ & $3.9 \times 10^{2}$ \\
\hline
\end{tabular}

Table 2 
Mean freq. $\max . \pm \quad$ Mean freq. $\min . \pm$ SD $\quad$ Mean duration \pm SD $\begin{array}{llll}\text { Animal } & \text { Sex } & \text { SD (CV) } \mathrm{kHz} & \text { (CV) } \mathrm{kHz}\end{array}$

\begin{tabular}{|c|c|c|c|c|}
\hline FB3 & $\bar{F}$ & $27.30 \pm 1.87(0.07)$ & $13.33 \pm 0.53(0.04)$ & $2.3 \pm 0.69(0.3)$ \\
\hline FB7 & $\mathrm{F}$ & $12.86 \pm 0.48(0.04)$ & $4.21 \pm 0.30(0.07)$ & $1.3 \pm 0.23(0.18)$ \\
\hline FB9 & $\mathrm{F}$ & $11.21 \pm 0.53(0.05)$ & $6.24 \pm 0.41(0.06)$ & $0.8 \pm 0.15(0.19)$ \\
\hline FB11 & $\mathrm{F}$ & $23.50 \pm 0.78(0.03)$ & $5.86 \pm 0.20(0.03)$ & $1.3 \pm 0.47(0.37)$ \\
\hline FB20 & M & $11.60 \pm 1.40(0.12)$ & $5.90 \pm 0.49(0.08)$ & $1.2 \pm 0.55(0.45)$ \\
\hline FB24 & M & $13.43 \pm 1.55(0.12)$ & $5.22 \pm 0.88(0.17)$ & $0.9 \pm 0.16(0.18)$ \\
\hline FB25 & $\mathrm{F}$ & $22.17 \pm 3.55(0.16)$ & $7.18 \pm 0.18(0.03)$ & $1 \pm 0.3(0.31)$ \\
\hline FB35 & $\mathrm{F}$ & $15.07 \pm 1.98(0.13)$ & $5.43 \pm 0.51(0.09)$ & $0.9 \pm 0.22(0.24)$ \\
\hline FB38 & M & $14.95 \pm 1.01(0.07)$ & $5.31 \pm 0.28(0.05)$ & $0.7 \pm 0.19(0.28)$ \\
\hline$*$ & & 14.81 & 5.15 & 0.1 \\
\hline$*$ & & 14.68 & 5.65 & 0.2 \\
\hline FB48 & M & $14.42 \pm 0.30(0.02)$ & $4.14 \pm 0.91(0.22)$ & $0.9 \pm 0.34(0.39)$ \\
\hline$*$ & & 9.29 & 5.27 & 0.8 \\
\hline$*$ & & 8.53 & 6.40 & 0.2 \\
\hline$*$ & & 7.03 & 6.02 & 0.1 \\
\hline$*$ & & 9.54 & 5.15 & 0.9 \\
\hline$*$ & & 10.67 & 7.28 & 0.8 \\
\hline FB54 & $\mathrm{F}$ & $21.46 \pm 3.65(0.17)$ & $6.20 \pm 0.57(0.09)$ & $1.2 \pm 0.37(0.31)$ \\
\hline$*$ & & 15.06 & 5.40 & 0.1 \\
\hline FB55 & $\mathrm{F}$ & $14.97 \pm 0.77(0.05)$ & $4.35 \pm 1.08(0.25)$ & $0.9 \pm 0.26(0.29)$ \\
\hline
\end{tabular}




\begin{tabular}{|c|c|c|c|c|}
\hline$*$ & & 14.85 & 6.40 & 0.1 \\
\hline FB67 & $\mathrm{F}$ & $23.02 \pm 2.04(0.09)$ & $4.99 \pm 0.19(0.04)$ & $2 \pm 0.38(0.19)$ \\
\hline FB84 & $\mathrm{F}$ & $19.47 \pm 1.76(0.09)$ & $6.58 \pm 0.31(0.05)$ & $1.2 \pm 0.33(0.27)$ \\
\hline FB90 & $\mathrm{F}$ & $24.68 \pm 2.00(0.08)$ & $3.31 \pm 0.70(0.21)$ & $1.2 \pm 0.1(0.08)$ \\
\hline FB97 & $\mathrm{F}$ & $12.50 \pm 0.28(0.02)$ & $7.00 \pm 0.45(0.06)$ & $1.2 \pm 0.35(0.3)$ \\
\hline FB101 & $\mathrm{F}$ & $15.68 \pm 4.51(0.29)$ & $4.09 \pm 0.88(0.21)$ & $0.8 \pm 0.45(0.53)$ \\
\hline FB105 & $\mathrm{F}$ & $11.56 \pm 2.40(0.21)$ & $4.76 \pm 0.42(0.09)$ & $0.5 \pm 0.19(0.35)$ \\
\hline FB118 & M & $17.55 \pm 1.31(0.07)$ & $6.73 \pm 0.66(0.10)$ & $1 \pm 0.42(0.41)$ \\
\hline FB122 & M & $14.21 \pm 0.26(0.02)$ & $5.28 \pm 1.66(0.31)$ & $0.8 \pm 0.16(0.2)$ \\
\hline FB138 & M & $20.74 \pm 1.54(0.07)$ & $10.09 \pm 0.28(0.03)$ & $1.7 \pm 0.36(0.21)$ \\
\hline FB140 & M & $18.62 \pm 0.71(0.04)$ & $4.09 \pm 0.55(0.13)$ & $1.8 \pm 0.67(0.37)$ \\
\hline FB146 & M & $15.40 \pm 1.42(0.09)$ & $6.11 \pm 1.21(0.20)$ & $1.1 \pm 0.36(0.32)$ \\
\hline FB151 & $\mathrm{F}$ & $15.34 \pm 2.10(0.14)$ & $5.23 \pm 0.66(0.13)$ & $0.7 \pm 0.14(0.2)$ \\
\hline$*$ & & 9.41 & 6.15 & 0.3 \\
\hline FB163 & $\mathrm{F}$ & $25.36 \pm 1.72(0.07)$ & $3.62 \pm 0.68(0.19)$ & $1.3 \pm 0.44(0.33)$ \\
\hline$*$ & & 9.54 & 3.39 & 0.5 \\
\hline$*$ & & 15.18 & 2.13 & 0.9 \\
\hline FB166 & M & $12.34 \pm 2.01(0.16)$ & $3.65 \pm 0.91(0.25)$ & $1.1 \pm 0.36(0.34)$ \\
\hline FB186 & M & $22.65 \pm 1.86(0.08)$ & $4.26 \pm 0.16(0.04)$ & $0.7 \pm 0.25(0.35)$ \\
\hline FB220 & M & $9.34 \pm 0.35(0.04)$ & $3.01 \pm 0.32(0.11)$ & $1 \pm 0.21(0.21)$ \\
\hline
\end{tabular}

438

439 Table 3 


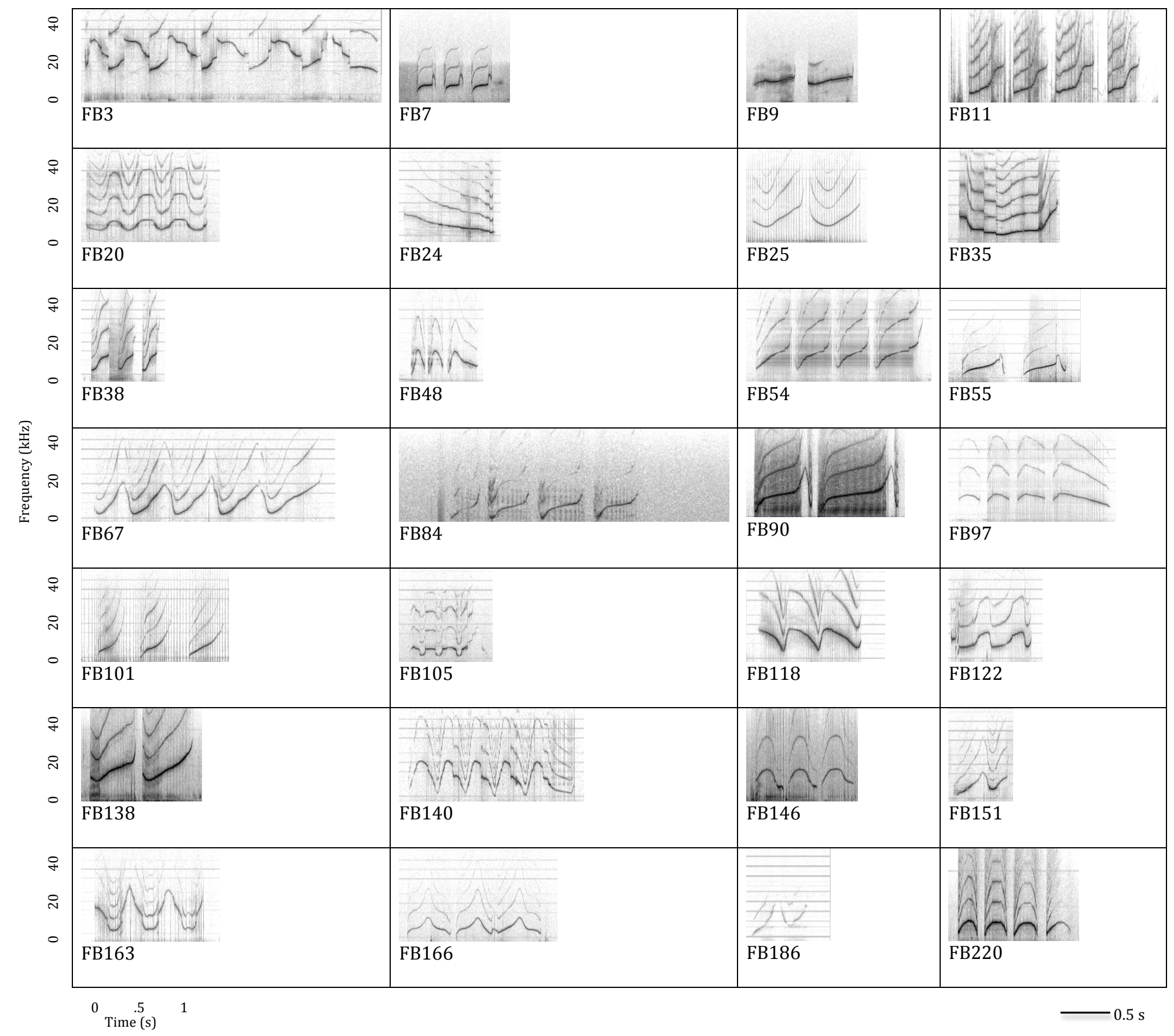



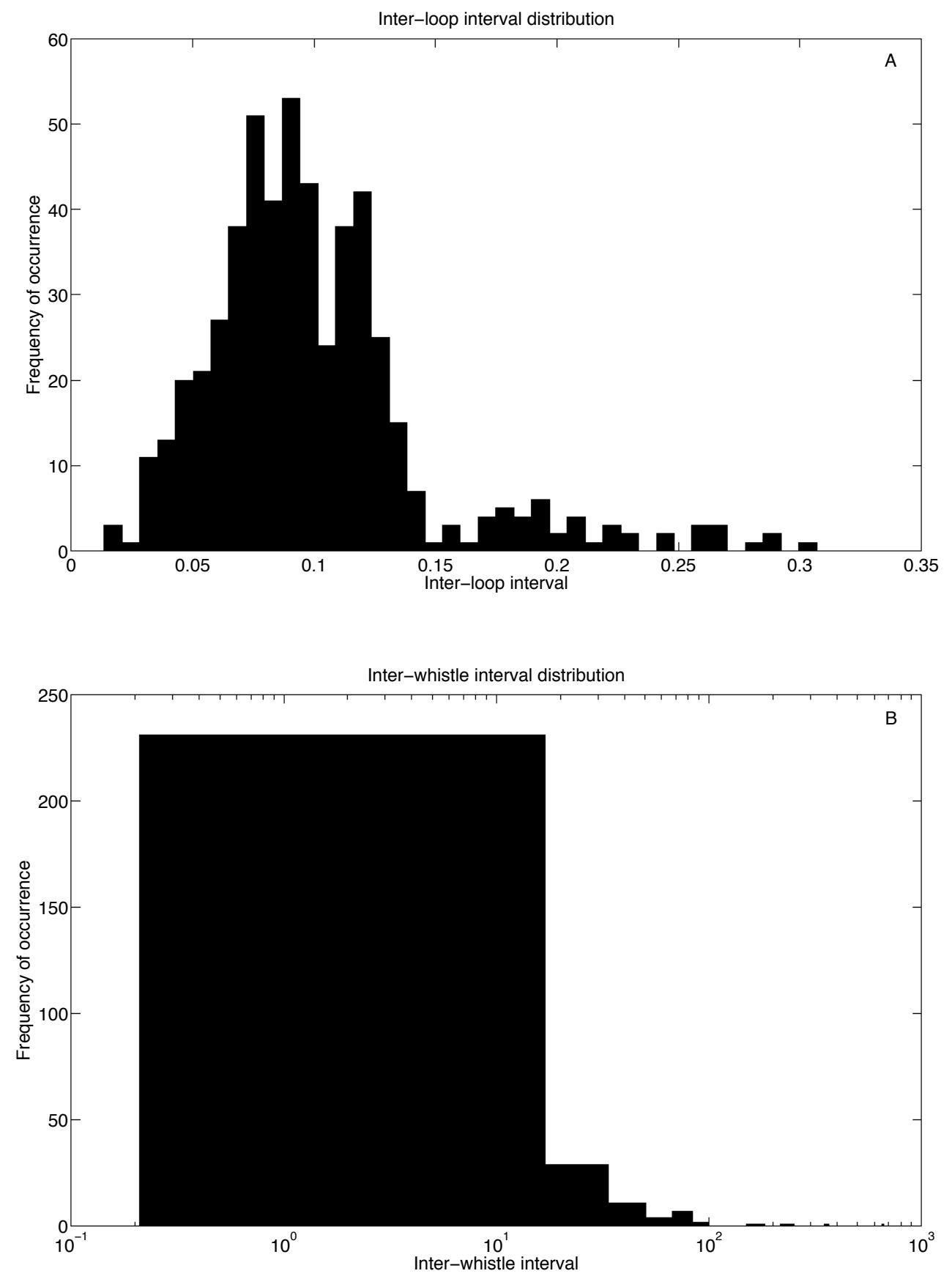